Supporting Information

\title{
Anderson Localization Enabled Spectrally \\ Stable Deep-Ultraviolet Laser Based on \\ Metallic Nanoparticles Decorated \\ AlGaN Multiple Quantum Wells
}

Meng-Jer Wu, Shang-Cheng Wu, Tien-Lin Shen, Yu-Ming Liao, Yang-Fang Chen*

Department of Physics, National Taiwan University, Taipei 10617, Taiwan

*E-mail:yfchen@phys.ntu.edu.tw

Keywords: Anderson localization, stabilized deep ultraviolet laser, surface plasmon resonance, random laser, $\mathrm{AlGaN}$ 


\section{Contents:}

A: Laser spectrum with and without metal nanoparticles.

B: PFT analysis

C: Exclusion of the possibility of Fabry-Perot resonance

D: Top surface optical microscope emission pattern image of the device under

$266 \mathrm{~nm}$ pulsed laser excitation

E: Configuration of the PL measurements 


\section{A: Laser spectrum with and without metal nanoparticles.}

To further explore the influence of aluminum particles in our devices, Figure S1 shows the laser spectrum with and without metal nanoparticles. The sample with aluminum nanoparticles can successfully evoke the laser action under the excitation, not the other way.

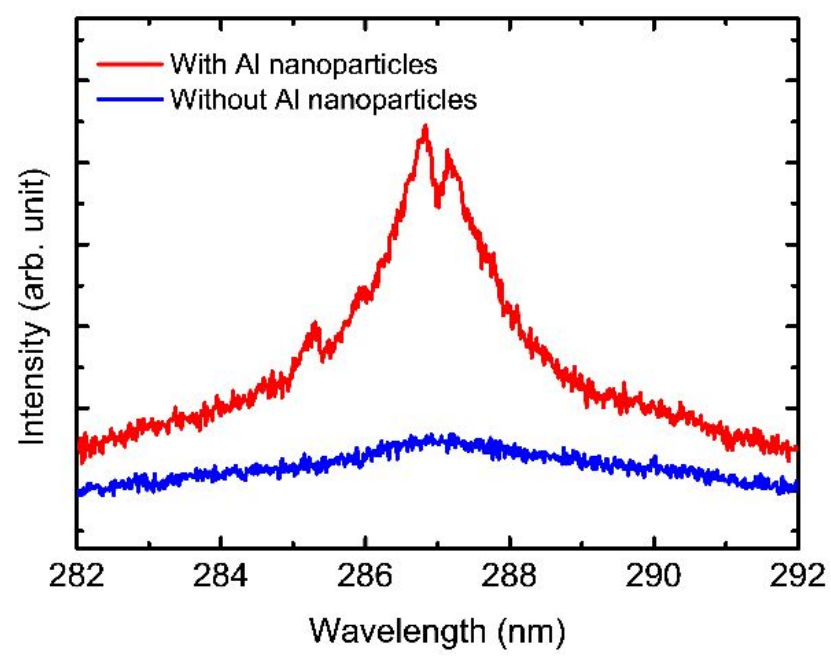

Figure S1. Laser spectrum with and without metal nanoparticles. 


\section{B: PFT analysis}

The power Fourier Transformation (PFT) intensity-frequency relation graph is shown in Figure R1. The PFT spectrum exhibits peaks at Fourier components $\mathrm{P}_{m}=\mathrm{mnL}_{c} / \pi$, where $\mathrm{m}$ is the order of the Fourier harmonic, $\mathrm{n}$ the refraction index of the gain medium (3.0), and $\mathrm{L}_{c}$ the cavity length. ${ }^{1-3}$ Through the relation between the Fourier peaks and the optical cavity length, we have calculated the optical cavity length, which is demonstrated in the insert of the figure. We have found that the mean optical cavity length of the device is around $11.01 \mu \mathrm{m}$, while the deviation of this statistical result is $2.11 \mu \mathrm{m}$. By comparing the cavity length and the thickness of device, we find that most of the close-loop resonance path should be horizontal to the top surface. In addition, since metal nanoparticles play an important role in the localization, the close-loop resonance path should close to the top surface, which further confirms the illustration shown in Figure S2. 


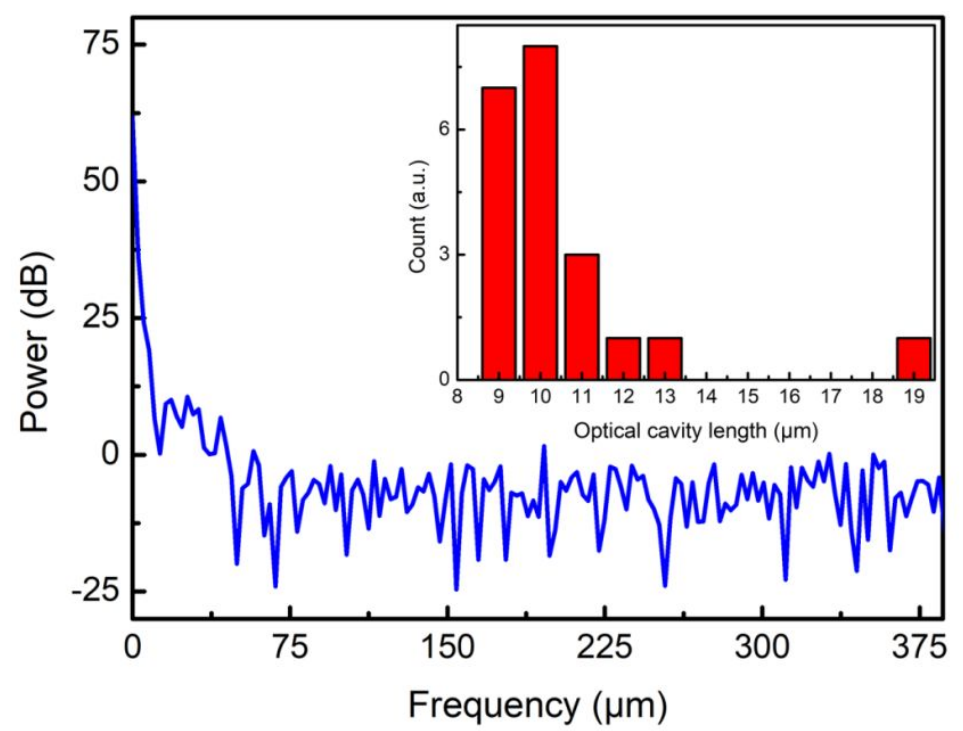

Figure S2. The power Fourier Transformation intensity-frequency relation graph. 


\section{C: Exclusion of the possibility of Fabry-Perot resonance}

As for the shape of the AlGaN multiple quantum wells, it is demonstrated in Figure R3. It should be noted that the figure only shows a part of the multiple quantum wells for the sake of illustrating its periodic structure. As shown in the figure, the variation of the band structure is vertical to the top surface of the device, which might be able to form a Fabry-Perot cavity. The peaks might be able to be attributed to Fabry-Perot modes if the spectra shown in the manuscript were measured when the emission were collected from the top surface instead of side wall. However, the emitted photons were collected from the side wall during the experiments. Therefore, the possibility of FabryPerot modes can be excluded from this case. 
D: Top surface optical microscope emission pattern image of the device under

$266 \mathrm{~nm}$ pulsed laser excitation

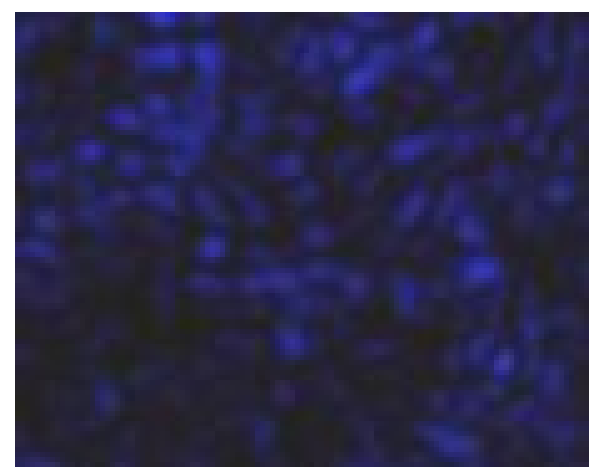

Figure S3. Top surface optical microscope emission pattern image of the device under $266 \mathrm{~nm}$ pulsed laser excitation. 


\section{E: Configuration of the PL measurements}

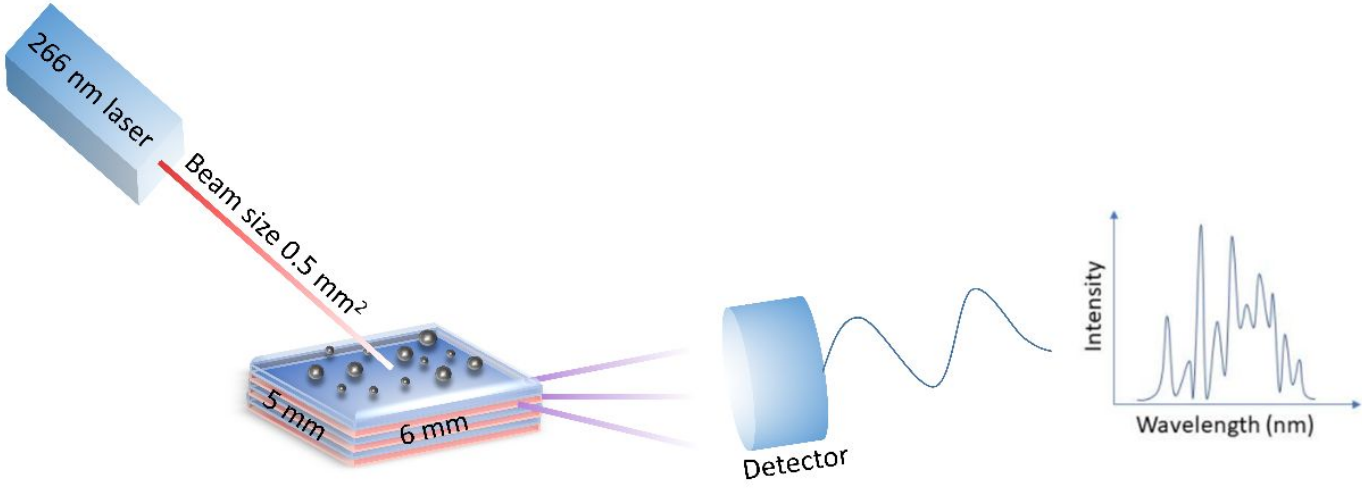

Figure S4. Configuration of the PL measurements. 
Reference:

1. Bian, Y.; Shi, X.; Hu, M.; Wang, Z. A Ring-Shaped Random Laser in Momentum Space. Nanoscale 2020, 12, 3166-3173.

2. Hu, Z.; Zhang, Q.; Miao, B.; Fu, Q.; Zou, G.; Chen, Y.; Luo, Yi.; Zhang, D.; Wang, P.; Ming, H.; Zhang, Q. Coherent Random Fiber Laser Based on Nanoparticles Scattering in the Extremely Weakly Scattering Regime. Phys. Rev. Lett. 2012, 109, 253901

3. Polson, R. C.; Vardeny, Z. V. Random Lasing in Human Tissues. Appl. Phys. Lett. 2004, 85, 1289-1291. 